\title{
SATURATED PROPERTIES PREDICTION IN CRITICAL REGION BY A QUARTIC EQUATION OF STATE
}

\author{
Yong Wang*, Guo-bo Huang and Chang-hua Ge \\ School of Pharmaceutical and Chemical Engineering, Taizhou University, Linhai 317000, P. R. \\ China
}

(Received June 8, 2010; revised September 13, 2010)

\begin{abstract}
A diverse substance library containing extensive PVT data for 77 pure components was used to critically evaluate the performance of a quartic equation of state and other four famous cubic equations of state in critical region. The quartic EOS studied in this work was found to significantly superior to the others in both vapor pressure prediction and saturated volume prediction in vicinity of critical point.
\end{abstract}

KEY WORDS: Equation of state, Saturated properties, Critical region

\section{INTRODUCTION}

Equation of state (EOS) is an important tool to describe properties of fluids. vdW type cubic EOS, such as the EOS proposed by Redlich-Kwong [1], Soave [2], and Peng-Robinson [3], received great attention and are used widely in calculating fluid thermodynamic properties for their simple form, few parameters, analytical solutions. However, due to their simplified forms, most of these famous EOS cannot describe simultaneously all the properties accurately, especially in the immediate vicinity of the critical point.

Critical properties are important for pure chemical substances, and they are indispensable in describing fluid phase behavior, predicting physical properties, developing equation of state (EOS), and so on. As the density difference between the equilibrium phases becomes very small on approaching the critical point, and owing to difficulties in adjusting the system temperature with a temperature step smaller than $1 \mathrm{~K}$, lots of researchers failed to measure the properties in critical region directly. No cubic equation gives accurate predictions of these properties in the critical region. Accuracy in the representation of saturated liquid densities in the critical region always leads to a loss of accuracy in the representation of saturated vapor densities and vice versa, concluded by Schmidt and Wenzel [4] several years ago. In recent years, researchers did lots of work to improve the accuracy of fluids properties prediction and the application in the near-critical region with EOS's. We think that the EOS of Duan et al. [5] is quite successful for supercritical fluids in this respect, but not in the sub-critical range, where phase equilibrium is very important. Kiselev et al. [6] developed a generalized cubic (GC) EOS for pure fluids based on the crossover sine model, which incorporates non-analytic scaling laws in the critical region. However, the GC EOS contains 10 adjustable parameters and an obscure theory, and it appears that in some cases the GC EOS can give non-physical behavior when extrapolated to high temperatures and densities (although modified later, such shortcoming still exist). Sukbae Lee $e t$ al. [7] suggested a newly constructed equation for fluid states, the generalized van der Waals (GvdW) EOS with the highly simplified Dieterici's form $P=[\mathrm{R} T /(V-b)]-a(A / V)(c)$ by a new model potential construction describing intermolecular interactions. They gave parameters for 12 pure normal small molecules fluids. With these parameters, it describes thermodynamic critical values well, but when extrapolated to other fluids, the accuracy declines.

On the other hand, Martin [8] pointed out that experimental data of fluids tend to follow quartic equation, and lots of quartic EOS were proposed since eighties of the last century [9-12].

*Corresponding author. E-mail: wangyong@tzc.edu.cn 
Yun et al. [13] proposed a quartic EOS (YSL EOS), which was constructed by a combination of the repulsive term of CCOR EOS [14] and the attractive term of PT [15] EOS. As shown by Yun et al., the quartic EOS can be used in both vapor and liquid phases, and it is capable of calculating a variety of fluids including polar and non-polar fluids. It is known and recognized nowadays that a cubic equation of state should not be expected to reproduce all thermodynamic properties accuracy, especially in critical region, partly because the cubic EOS usually has a constant value of compressibility factor, such as the PR EOS which has its value of compressibility factor being 0.307. Different from PR EOS, however, the YSL EOS has an adjustable compressibility factor which can reproduce the experimental value. The purpose of this work is to investigate the prediction accuracy of the saturated properties in the critical region by the YSL EOS, and four famous cubic EOS, SRK, PR, CCOR, and PT were used for comparison, and, on the other hand, to provide references of selection of EOS in critical region for later researchers after comparative analysis.

\section{RESULTS AND DISCUSSION}

\section{Comparison of calculation accuracy of saturated vapor pressure}

There are a large number of requirements for a good EOS. Good prediction accuracy of vapor pressure is the primary criterion for evaluation of an EOS, since the accurate prediction of vapor pressure is a prerequisite of the exact representation of phase equilibria in fluid mixers. Table 1 lists the calculated results by YSL EOS, as well as those by SRK, PR, PT, and CCOR. The experimental data of pure substances used in this work are all taken from Smith [16] and Vargaftik [17]. From the comparison of deviations between experimental and calculated saturated vapor pressures in Table 1, we can see that vapor pressure predictions accuracy of these EOS in the calculation temperature range were all considerable. This is because parameters in most of the vdW type EOS are determined by fitting calculated data of vapor pressure to experimental data around $T_{r}=0.7$ in articles to optimize the representation of vapor pressures.

Table 1A. Calculated results of vapor pressures by YSL EOS, as well as those by SRK, PR, PT, and CCOR.

\begin{tabular}{|c|c|c|c|c|c|c|c|c|c|c|}
\hline \multirow[t]{3}{*}{ No. } & \multirow[t]{3}{*}{ Substance } & \multirow[t]{3}{*}{$T_{\mathrm{r}}$} & \multirow[t]{3}{*}{$N_{\mathrm{p}}$} & \multicolumn{7}{|c|}{$\mathrm{AARD}^{*}$} \\
\hline & & & & \multicolumn{3}{|c|}{ YSL } & & \multirow{2}{*}{$\frac{\text { SRK }}{v^{v}}$} & \multirow[b]{2}{*}{$v^{1}$} & \multirow[b]{2}{*}{$p^{\mathrm{s}}$} \\
\hline & & & & $p^{\mathrm{s}}$ & $v^{\mathrm{v}}$ & $v^{1}$ & $p^{\mathrm{s}}$ & & & \\
\hline 1 & Methane & $0.71-0.99$ & 10 & 0.39 & 2.24 & 0.95 & 0.87 & 0.79 & 11.17 & 0.63 \\
\hline 2 & Ethane & $0.82-0.99$ & 6 & 0.53 & 1.81 & 2.59 & 1.03 & 1.05 & 15.57 & 0.43 \\
\hline 3 & Propane & $0.75-0.99$ & 9 & 1.75 & 4.55 & 2.08 & 0.74 & 1.57 & 13.87 & 0.52 \\
\hline 4 & Butane & $0.81-0.99$ & 7 & 0.55 & 2.05 & 1.02 & 1.38 & 1.57 & 14.09 & 0.56 \\
\hline 5 & Isobutane & $0.85-0.99$ & 5 & 0.52 & 3.75 & 2.77 & 1.26 & 4.01 & 13.19 & 0.56 \\
\hline 6 & Pentane & $0.77-0.99$ & 8 & 4.43 & 12.01 & 1.01 & 7.70 & 12.72 & 13.05 & 8.02 \\
\hline 7 & Hexane & $0.86-0.99$ & 5 & 1.05 & 4.18 & 0.71 & 2.04 & 2.03 & 13.65 & 1.23 \\
\hline 8 & 2-Methylpentane & $0.84-0.99$ & 5 & 0.68 & 4.11 & 3.18 & 1.18 & 1.65 & 19.37 & 0.53 \\
\hline 9 & 3-Methylpentane & $0.90-0.99$ & 3 & 0.17 & 3.52 & 1.25 & 1.55 & 1.40 & 24.07 & 0.94 \\
\hline 10 & 2,2-Dimethylbutane & $0.88-0.99$ & 4 & 0.49 & 4.24 & 0.30 & 0.88 & 2.39 & 18.51 & 0.22 \\
\hline 11 & Heptane & $0.86-0.99$ & 5 & 0.71 & 9.32 & 0.70 & 2.42 & 8.50 & 23.9 & 1.57 \\
\hline 12 & 2-Methylhexane & $0.89-0.99$ & 4 & 0.92 & 3.57 & 1.53 & 1.13 & 1.82 & 25.63 & 0.45 \\
\hline 13 & 3-Methylhexane & $0.90-0.99$ & 3 & 0.65 & 3.86 & 0.49 & 1.02 & 2.45 & 27.51 & 0.36 \\
\hline 14 & 3-Ethylpentane & $0.85-0.99$ & 5 & 0.57 & 3.59 & 0.61 & 1.20 & 1.56 & 19.64 & 0.36 \\
\hline 15 & 2,2-Dimethylpentane & $0.85-0.99$ & 5 & 0.75 & 3.88 & 0.25 & 1.04 & 1.74 & 20.60 & 0.23 \\
\hline 16 & 2,3-Dimethylpentane & $0.87-0.99$ & 4 & 0.83 & 3.71 & 0.74 & 0.88 & 2.46 & 20.74 & 0.15 \\
\hline 17 & 2,4-Dimethylpentane & $0.90-0.99$ & 3 & 0.74 & 3.66 & 1.03 & 0.93 & 2.29 & 23.84 & 0.27 \\
\hline
\end{tabular}

Bull. Chem. Soc. Ethiop. 2011, 25(2) 


\begin{tabular}{|c|c|c|c|c|c|c|c|c|c|c|}
\hline 18 & 3,3-Dimethylpentane & $0.90-0.99$ & 3 & 0.43 & 3.08 & 1.72 & 1.28 & 1.58 & 18.40 & 0.66 \\
\hline 19 & 2,2,3-Trimethylbutane & $0.90-0.99$ & 4 & 0.7 & 3.17 & -- & 1.18 & 1.81 & -- & 0.55 \\
\hline 20 & Octane & $0.87-0.99$ & 5 & 0.67 & 9.78 & 1.00 & 2.13 & 6.34 & 25.9 & 1.31 \\
\hline 21 & 2-Methylheptane & $0.87-0.99$ & 4 & 1.01 & 3.90 & 0.72 & 1.34 & 1.52 & 25.40 & 0.56 \\
\hline 22 & 3-Methylheptane & $0.88-0.99$ & 4 & 1.41 & 7.64 & 0.56 & 1.54 & 1.56 & 21.48 & 0.77 \\
\hline 23 & 4-Methylheptane & $0.88-0.99$ & 4 & 1.87 & 5.14 & -- & 1.20 & 1.85 & -- & 0.44 \\
\hline 24 & 3-Ethyhexane & $0.88-0.99$ & 4 & 0.27 & 2.49 & 0.19 & 2.49 & 1.87 & 30.92 & 1.74 \\
\hline 25 & 2,2-Dimethylhexane & $0.88-0.99$ & 4 & 1.32 & 4.49 & 0.29 & 1.00 & 2.24 & 19.87 & 0.25 \\
\hline 26 & 2,3-Dimethylhexane & $0.88-0.99$ & 4 & 1.45 & 4.52 & 1.17 & 0.93 & 2.24 & 24.23 & 0.21 \\
\hline 27 & 2,4-Dimethylhexane & $0.88-0.99$ & 4 & 1.84 & 5.10 & 0.93 & 0.90 & 2.35 & 26.9 & 0.16 \\
\hline 28 & 2,5-Dimethylhexane & $0.88-0.99$ & 4 & 0.58 & 3.71 & -- & 1.25 & 1.92 & -- & 0.49 \\
\hline 29 & 3,3-Dimethylhexane & $0.88-0.99$ & 4 & 1.06 & 7.93 & -- & 1.17 & 2.04 & -- & 0.42 \\
\hline 30 & 3-Ethyl-2-methylpentane & $0.88-0.99$ & 4 & 0.1 & 4.06 & -- & 1.2 & 1.60 & -- & 0.75 \\
\hline 31 & 3-Ethyl-3-methylpentane & $0.88-0.99$ & 4 & 0.55 & 3.85 & 1.16 & 1.35 & 1.56 & 20.48 & 0.61 \\
\hline 32 & 3,4-Dimethylhexane & $0.88-0.99$ & 4 & 2.62 & 3.84 & -- & 2.98 & 1.69 & -- & 2.23 \\
\hline 33 & 2,2,3-Trimethylpentane & $0.88-0.99$ & 4 & 0.58 & 8.34 & 3.55 & 1.30 & 1.56 & 26.90 & 0.56 \\
\hline 34 & 2,2,4-Trimethypentane & $0.86-0.99$ & 5 & 0.52 & 3.54 & 0.69 & 1.57 & 1.25 & 18.10 & 0.74 \\
\hline 35 & Cyclopropane & $0.86-0.99$ & 5 & 1.28 & 2.25 & 0.49 & 1.31 & 2.11 & 18.99 & 0.72 \\
\hline 36 & 2,3,3-Trimethylpentane & $0.88-0.99$ & 4 & 0.76 & 3.66 & -- & 1.14 & 1.85 & -- & 0.71 \\
\hline 37 & 2,3,4-Trimethylpentane & $0.87-0.99$ & 5 & 0.74 & 3.51 & 1.18 & 1.27 & 1.58 & 21.44 & 0.48 \\
\hline 38 & Cyclopentane & $0.86-0.99$ & 5 & 0.56 & 3.34 & 2.29 & 0.98 & 1.86 & 18.43 & 0.29 \\
\hline 39 & Cyclohexane & $0.89-0.98$ & 3 & 0.47 & -- & 6.96 & 0.97 & -- & 20.05 & 0.31 \\
\hline 40 & Methylcyclopentane & $0.89-0.99$ & 4 & 0.57 & 2.36 & -- & 1.16 & 1.66 & -- & 0.52 \\
\hline 41 & Methylcyclohexane & $0.85-0.99$ & 5 & 0.42 & 4.33 & 0.72 & 1.18 & 1.51 & 19.98 & 0.41 \\
\hline 42 & Ethylcyclopentane & $0.89-0.99$ & 4 & 1.43 & 4.90 & 0.67 & 0.61 & 3.27 & 19.56 & 0.06 \\
\hline 43 & Ethene & $0.86-0.99$ & 5 & 0.75 & 2.60 & 1.27 & 0.86 & 0.68 & 16.84 & 0.33 \\
\hline 44 & Propene & $0.86-0.99$ & 5 & 0.1 & 1.58 & 7.73 & 0.59 & 3.99 & 21.34 & 0.37 \\
\hline 45 & Cycloheptane & 0.89-0.99 & 4 & 1.79 & 2.01 & -- & 3.15 & 7.30 & -- & 3.77 \\
\hline 46 & Butene & $0.88-0.99$ & 4 & 0.15 & 1.56 & -- & 1.11 & 1.52 & -- & 0.54 \\
\hline 47 & Pentene & $0.89-0.99$ & 4 & 0.43 & 2.49 & 2.18 & 1.58 & 2.01 & 21.69 & 0.94 \\
\hline 48 & 1,3-Butadiene & $0.87-0.99$ & 4 & 0.3 & 2.68 & -- & 2.03 & 1.45 & -- & 1.3 \\
\hline 49 & cis-2-Pentene & $0.83-0.99$ & 6 & 0.82 & 2.71 & -- & 2.33 & 2.34 & -- & 1.85 \\
\hline 50 & Benzene & $0.89-0.99$ & 4 & 0.67 & 1.02 & -- & 0.58 & 4.62 & -- & 1.17 \\
\hline 51 & Propyne & $0.89-0.99$ & 4 & 0.31 & 1.80 & 3.98 & 1.22 & 0.58 & 19.59 & 0.59 \\
\hline 52 & Toluene & $0.85-0.99$ & 5 & 1.33 & 4.85 & -- & 1.62 & 1.39 & -- & 0.81 \\
\hline 53 & Acetone & $0.86-0.99$ & 5 & 0.5 & 2.57 & 1.86 & 1.50 & 1.71 & 38.30 & 0.71 \\
\hline 54 & m-Xylene & $0.87-0.99$ & 5 & 1.23 & 4.52 & -- & 1.47 & 1.50 & -- & 0.67 \\
\hline 55 & Chlorodifluoromethane & $0.88-0.98$ & 4 & 1.75 & 2.91 & 1.40 & 1.32 & 1.44 & 24.08 & 0.63 \\
\hline 56 & Chloromethane & $0.87-0.99$ & 4 & 0.12 & 1.70 & 1.50 & 0.69 & 3.4 & 24.73 & 0.12 \\
\hline 57 & Dichlorodifluoromethane & $0.87-0.99$ & 4 & 0.43 & 2.52 & 2.27 & 1.18 & 8.06 & 17.17 & 0.51 \\
\hline 58 & $\mathrm{C}_{2} \mathrm{Cl}_{2} \mathrm{~F}_{4}$ & $0.80-0.99$ & 7 & 0.76 & 4.55 & -- & 0.31 & 5.28 & -- & 0.43 \\
\hline 59 & Trichlorofluoromethane & $0.87-0.99$ & 5 & 0.52 & 1.36 & 2.94 & 0.67 & 1.87 & 18.07 & 0.08 \\
\hline 60 & Trafluoromethane & $0.87-0.99$ & 4 & 0.71 & 2.12 & -- & 0.45 & 6.82 & -- & 0.20 \\
\hline 61 & $\mathrm{C}_{2} \mathrm{CL}_{3} \mathrm{~F}_{3}$ & $0.89-0.99$ & 4 & 0.8 & 2.39 & 2.09 & 0.54 & 7.75 & 21.01 & 0.12 \\
\hline 62 & Hexafluoethane & $0.81-0.99$ & 6 & 0.32 & -- & -- & 0.97 & -- & -- & 0.38 \\
\hline 63 & Octafluoropropane & $0.81-0.99$ & 7 & 0.23 & 1.13 & 4.54 & 0.96 & 2.34 & 15.39 & 0.36 \\
\hline 64 & 1-Propanol & $0.83-0.97$ & 5 & 2.32 & 2.92 & 10.03 & 1.74 & 5.66 & 24.45 & 2.51 \\
\hline 65 & Butanol & $0.85-0.98$ & 5 & 1.97 & 1.02 & 0.58 & 2.11 & 6.36 & 23.58 & 2.89 \\
\hline 66 & Ethanol & $0.85-0.99$ & 5 & 0.98 & 3.18 & 1.19 & 1.88 & 0.83 & 26.99 & 1.20 \\
\hline 67 & Water & $0.87-0.99$ & 4 & 1.37 & 3.57 & -- & 3.02 & 3.58 & -- & -- \\
\hline 68 & Ammonia & $0.81-0.99$ & 7 & 1.46 & -- & 2.38 & 0.36 & -- & -- & 0.33 \\
\hline 69 & Neon & $0.80-0.99$ & 7 & 0.67 & 4.89 & 3.22 & 1.26 & 7.16 & 8.44 & 1.56 \\
\hline 70 & Argon & $0.82-0.99$ & 6 & 0.8 & 1.02 & 1.74 & 0.66 & 3.16 & 14.71 & 0.45 \\
\hline 71 & Carbon monoxide & $0.86-0.99$ & 5 & 0.6 & 1.48 & 7.34 & 1.13 & 11.68 & 8.09 & 1.60 \\
\hline 72 & Carbon dioxide & $0.87-0.99$ & 4 & 0.22 & 4.37 & 3.31 & 0.23 & 7.75 & 27.07 & 0.11 \\
\hline 73 & Nitrogen & $0.85-0.99$ & 5 & 1.18 & 3.64 & 3.90 & 0.14 & 2.53 & 18.08 & 0.04 \\
\hline
\end{tabular}

Bull. Chem. Soc. Ethiop. 2011, 25(2) 


\begin{tabular}{|c|l|c|c|c|c|c|c|c|c|c|}
\hline 74 & Fluorine & $0.80-0.99$ & 7 & 2.78 & 3.52 & 1.04 & 3.91 & 10.06 & 11.32 & 4.20 \\
\hline 75 & Oxygen & $0.85-0.99$ & 5 & 0.9 & 2.47 & 2.80 & 0.37 & 2.02 & 16.90 & 0.541 \\
\hline 76 & Sulfur dioxide & $0.85-0.99$ & 5 & 0.18 & 6.04 & 2.16 & 0.18 & 17.62 & 25.35 & 0.19 \\
\hline 77 & Chlorine & $0.84-0.99$ & 6 & 0.96 & 4.93 & 3.42 & 0.34 & 8.29 & 25.56 & 1.16 \\
\hline & Average & & & 0.90 & 3.71 & 2.07 & 1.33 & 3.39 & 20.42 & 0.86 \\
\hline
\end{tabular}

$* \mathrm{AARD}=\sum\left|\left(X_{\exp }-X_{c a l}\right) / X_{\exp }\right| / N_{p} \times 100, \mathrm{X}=p^{\mathrm{s}}, v^{l}, v^{\mathrm{v}} ; N_{\mathrm{p}}$ : number of data points.

Table 1B. Deviations of the saturated vapor pressure of some pure fluids predicted with different EOS.

\begin{tabular}{|c|c|c|c|c|c|c|c|c|c|c|c|}
\hline No. & Substance & $T_{\mathrm{r}}$ & $N_{\mathrm{p}}$ & \multicolumn{8}{|c|}{ AARD ${ }^{*}$} \\
\hline & & & & PR & & & PT & & & $\begin{array}{c}\mathrm{CCO} \\
\mathrm{R}\end{array}$ & \\
\hline & & & & $v^{\mathrm{v}}$ & $v^{1}$ & $p^{\mathrm{s}}$ & $v^{v}$ & $v^{1}$ & $p^{\mathrm{s}}$ & $v^{v}$ & $v^{1}$ \\
\hline 1 & Methane & $0.71-0.99$ & 10 & 2.71 & 6.43 & 1.36 & 1.51 & 8.99 & 0.74 & 10.71 & 3.32 \\
\hline 2 & Ethane & $0.82-0.99$ & 6 & 1.76 & 5.18 & 0.99 & 1.18 & 9.85 & 0.85 & 12.13 & 3.33 \\
\hline 3 & Propane & $0.75-0.99$ & 9 & 1.85 & 4.57 & 0.52 & 1.48 & 6.12 & 0.89 & 10.30 & 2.07 \\
\hline 4 & Butane & $0.81-0.99$ & 7 & 1.87 & 1.97 & 0.84 & 1.62 & 4.32 & 1.21 & 12.59 & 0.87 \\
\hline 5 & Isobutane & $0.85-0.99$ & 5 & 6.57 & 0.97 & 0.87 & 5.92 & 4.35 & 1.23 & 17.76 & 0.36 \\
\hline 6 & Pentane & $0.77-0.99$ & 8 & 12.14 & 3.02 & 8.00 & 12.13 & 5.01 & 7.90 & 9.45 & 3.45 \\
\hline 7 & Hexane & $0.86-0.99$ & 5 & 2.59 & 8.23 & 1.14 & 2.4 & 8.37 & 2.02 & 15.62 & 2.20 \\
\hline 8 & 2-Methylpentane & $0.84-0.99$ & 5 & 1.11 & 5.59 & 0.31 & 0.91 & 6.5 & 1.14 & 13.67 & 1.63 \\
\hline 9 & 3-Methylpentane & $0.90-0.99$ & 3 & 2.48 & 10.25 & 0.96 & 2.17 & 11.52 & 1.63 & 15.85 & 3.82 \\
\hline 10 & 2,2-Dimethylbutane & $0.88-0.99$ & 4 & 0.71 & 4.87 & 0.35 & 0.15 & 7.49 & 0.95 & 13.68 & 1.67 \\
\hline 11 & Heptane & $0.86-0.99$ & 5 & 10.52 & 9.65 & 1.31 & 10.57 & 8.13 & 2.28 & 23.01 & 1.98 \\
\hline 12 & 2-Methylhexane & $0.89-0.99$ & 4 & 1.31 & 11.52 & 0.32 & 1.37 & 10.74 & 1.11 & 15.65 & 3.24 \\
\hline 13 & 3-Methylhexane & $0.90-0.99$ & 3 & 0.81 & 13.21 & 0.26 & 0.88 & 12.64 & 1.02 & 15.22 & 4.90 \\
\hline 14 & 3-Ethylpentane & $0.85-0.99$ & 5 & 0.98 & 5.87 & 0.26 & 0.989 & 5.72 & 1.14 & 14.41 & 1.63 \\
\hline 15 & 2,2-Dimethylpentane & $0.85-0.99$ & 5 & 0.75 & 6.73 & 0.23 & 0.62 & 7.40 & 1.02 & 13.93 & 1.92 \\
\hline 16 & 2,3-Dimethylpentane & $0.87-0.99$ & 4 & 0.49 & 6.96 & 0.17 & 0.51 & 7.36 & 0.78 & 13.98 & 1.77 \\
\hline 17 & 2,4-Dimethylpentane & $0.90-0.99$ & 3 & 0.97 & 9.79 & 0.23 & 0.84 & 9.94 & 0.96 & 15.07 & 2.90 \\
\hline 18 & 3,3-Dimethylpentane & $0.90-0.99$ & 3 & 1.87 & 5.02 & 0.69 & 1.52 & 6.31 & 1.35 & 15.43 & 1.58 \\
\hline 19 & 2,2,3-Trimethylbutane & $0.90-0.99$ & 4 & 1.53 & -- & 0.67 & 1.16 & -- & 1.26 & 14.92 & -- \\
\hline 20 & Octane & $0.87-0.99$ & 5 & 8.42 & 11.46 & 0.88 & 8.56 & 8.27 & 1.82 & 21.65 & 1.78 \\
\hline 21 & 2-Methylheptane & $0.87-0.99$ & 4 & 1.41 & 11.07 & 0.26 & 1.59 & 8.58 & 1.13 & 15.77 & 1.86 \\
\hline 22 & 3-Methylheptane & $0.88-0.99$ & 4 & 1.49 & 14.74 & 0.46 & 1.65 & 12.47 & 1.37 & 15.82 & 4.36 \\
\hline 23 & 4-Methylheptan & $0.88-0.99$ & 4 & 1.18 & -- & 0.18 & 1.36 & -- & 1.03 & 15.67 & -- \\
\hline 24 & 3-Ethyhexane & $0.88-0.99$ & 4 & 1.16 & 9.99 & 1.49 & 1.32 & 13.4 & 2.36 & 15.5 & 4.08 \\
\hline 25 & 2,2-Dimethylhex & $0.88-0.99$ & 4 & 0.62 & 7.13 & 0.12 & 0.65 & 9.15 & 0.94 & 14.89 & 2.54 \\
\hline 26 & 2,3-Dimethylhexane & $0.88-0.99$ & 4 & 0.64 & 14.17 & 0.12 & 0.78 & 10.63 & 0.87 & 15.00 & 2.58 \\
\hline 27 & 2,4-Dimethylhexane & $0.88-0.99$ & 4 & 0.52 & 10.49 & 0.12 & 0.68 & 11.75 & 0.86 & 14.85 & 4.65 \\
\hline 28 & 2,5-Dimethylhexane & $0.88-0.99$ & 4 & 1.22 & -- & 0.25 & 1.53 & \begin{tabular}{|l|}
-- \\
\end{tabular} & 1.11 & 15.75 & -- \\
\hline 29 & 3,3-Dimethylhexane & $0.88-0.99$ & 4 & 1.36 & -- & 0.29 & 1.31 & -- & 1.14 & 15.34 & -- \\
\hline 30 & 3-Ethyl-2-methylpentane & $0.88-0.99$ & 4 & 1.41 & -- & 0.57 & 1.56 & -- & 1.63 & 15.58 & -- \\
\hline 31 & 3-Ethyl-3-methylpentane & $0.88-0.99$ & 4 & 1.60 & 8.15 & 0.78 & 1.98 & 10.76 & 1.36 & 15.36 & 1.42 \\
\hline 32 & 3,4-Dimethylhexane & $0.88-0.99$ & 4 & 1.24 & -- & 2.05 & 1.28 & -- & 2.92 & 15.42 & -- \\
\hline 33 & 2,2,3-Trimethylpentane & $0.88-0.99$ & 4 & 1.49 & 12.71 & 0.50 & 1.34 & 11.03 & 1.31 & 15.20 & 5.07 \\
\hline 34 & 2,2,4-Trimethypentane & $0.86-0.99$ & 5 & 1.85 & 5.72 & 0.64 & 1.69 & 5.81 & 1.53 & 15.15 & 1.78 \\
\hline 35 & Cyclopropane & $0.86-0.99$ & 5 & 5.62 & 5.59 & 1.12 & 3.28 & 11.83 & 1.29 & 16.61 & 5.27 \\
\hline 36 & 2,3,3-Trimethylpentane & $0.88-0.99$ & 4 & 1.71 & -- & 0.38 & 0.97 & \begin{tabular}{|l|}
-- \\
\end{tabular} & 1.16 & 14.81 & -- \\
\hline 37 & 2,3,4-Trimethylpentane & $0.87-0.99$ & 5 & 1.28 & 7.49 & 0.36 & 1.22 & 7.15 & 1.23 & 15.06 & 1.45 \\
\hline 38 & Cyclopentane & $0.86-0.99$ & 5 & 0.97 & 5.41 & 0.53 & 0.20 & 9.06 & 1.00 & 13.24 & 2.52 \\
\hline 39 & Cyclohexane & $0.89-0.98$ & 3 & -- & 6.98 & 0.50 & -- & 9.98 & 1.06 & -- & 2.68 \\
\hline 40 & Methylcyclopentane & 0.89-0.99 & 4 & 1.57 & -- & 0.63 & 0.98 & -- & 1.24 & 14.60 & -- \\
\hline 41 & Methylcyclohexane & $0.85-0.99$ & 5 & 1.14 & 6.26 & 0.54 & 0.76 & 8.72 & 1.18 & 13.81 & 2.75 \\
\hline 42 & Ethylcyclopentane & $0.89-0.99$ & 4 & 0.21 & 6.10 & 0.05 & 0.45 & 7.13 & 0.70 & 13.71 & 1.22 \\
\hline
\end{tabular}

Bull. Chem. Soc. Ethiop. 2011, 25(2) 


\begin{tabular}{|c|c|c|c|c|c|c|c|c|c|c|c|}
\hline 43 & Ethene & $0.86-0.99$ & 5 & 2.51 & 5.26 & 0.83 & 1.20 & 11.60 & 0.80 & 13.12 & 4.03 \\
\hline 44 & Propene & $0.86-0.99$ & 5 & 1.55 & 9.18 & 0.50 & 0.86 & 14.44 & 0.67 & 12.63 & 3.82 \\
\hline 45 & Cycloheptane & 0.89-0.99 & 4 & 3.93 & -- & 3.68 & 4.46 & \begin{tabular}{|l|}
-- \\
\end{tabular} & 3.08 & 9.87 & -- \\
\hline 46 & Butene & $0.88-0.99$ & 4 & 2.30 & -- & 0.77 & 1.21 & -- & 1.17 & 14.16 & -- \\
\hline 47 & Pentene & $0.89-0.99$ & 4 & 1.23 & 7.89 & 1.05 & 0.63 & 8.33 & 1.66 & 14.31 & 3.12 \\
\hline 48 & 1.3-Butadiene & $0.87-0.99$ & 4 & 2.12 & -- & 1.56 & 1.87 & -- & 2.05 & 14.17 & -- \\
\hline 49 & cis-2-Pentene & $0.83-0.99$ & 6 & 5.50 & -- & 1.89 & 4.95 & -- & 2.42 & 18.41 & -- \\
\hline 50 & Benzene & $0.89-0.99$ & 4 & 2.02 & -- & 1.14 & 2.37 & -- & 0.57 & 12.76 & -- \\
\hline 51 & Propyne & $0.89-0.99$ & 4 & 2.32 & 6.02 & 0.76 & 1.79 & 9.28 & 1.30 & 15.17 & .71 \\
\hline 52 & Toluene & $0.85-0.99$ & 5 & 1.92 & -- & 0.86 & 1.58 & -- & 1.62 & 14.72 & -- \\
\hline 53 & Acetone & $0.86-0.99$ & 5 & 1.56 & 22.47 & 0.61 & 1.57 & 22.44 & 1.48 & 15.17 & 15.37 \\
\hline 54 & $m$-Xylene & $0.87-0.99$ & 5 & 1.62 & -- & 0.51 & 1.57 & -- & 1.41 & 15.39 & -- \\
\hline 55 & Chlorodifluoromethane & $0.88-0.98$ & 4 & 2.32 & 10.41 & 0.85 & 1.94 & 14.14 & 1.38 & 14.87 & 6.37 \\
\hline 56 & Chloromethane & $0.87-0.99$ & 4 & 1.39 & 11.16 & 0.34 & 2.02 & 16.55 & 0.69 & 10.89 & 8.58 \\
\hline 57 & Dichlorodifluoromethane & $0.87-0.99$ & 4 & 5.44 & 4.52 & 0.82 & 5.98 & 8.50 & 1.19 & 13.17 & 2.04 \\
\hline 58 & $\mathrm{C}_{2} \mathrm{Cl}_{2} \mathrm{~F}_{4}$ & $0.80-0.99$ & 7 & 1.66 & -- & 0.37 & 2.19 & -- & 0.31 & 12.16 & -- \\
\hline 59 & Trichlorofluoromethane & $0.87-0.99$ & 5 & 4.69 & 5.20 & 0.27 & 3.96 & 8.98 & 0.69 & 16.46 & 2.26 \\
\hline 60 & Trafluoromethane & $0.87-0.99$ & 4 & 5.80 & -- & 0.09 & 6.06 & -- & 0.47 & 14.39 & -- \\
\hline 61 & $\mathrm{C}_{2} \mathrm{CL}_{3} \mathrm{~F}_{3}$ & 0.89-0.99 & 4 & 10.66 & 7.67 & 0.09 & 10.28 & 9.38 & 0.61 & 22.82 & 2.25 \\
\hline 62 & Hexafluoethane & $0.81-0.99$ & 6 & -- & -- & 0.744 & -- & -- & 1.06 & \begin{tabular}{|l|}
-- \\
\end{tabular} & -- \\
\hline 63 & Octafluoropropane & 0.81-0.99 & 7 & 1.11 & 3.33 & 0.25 & 1.32 & 2.64 & 0.96 & 15.71 & 5.90 \\
\hline 64 & 1-Propanol & $0.83-0.97$ & 5 & 4.24 & 10.01 & 4.24 & 4.99 & 3.10 & 4.90 & 7.51 & 4.78 \\
\hline 65 & Butanol & $0.85-0.98$ & 5 & 4.54 & 9.36 & 4.27 & 4.84 & 3.87 & 4.47 & 9.01 & 5.54 \\
\hline 66 & Ethanol & 0.85-0.99 & 5 & 1.61 & 20.48 & 0.88 & 2.50 & 8.84 & 1.06 & 14.27 & 1.77 \\
\hline 67 & Water & $0.87-0.99$ & 4 & -- & -- & 4.98 & -- & -- & 1.55 & \begin{tabular}{|l|}
-- \\
\end{tabular} & -- \\
\hline 68 & Ammonia & 0.81-0.99 & 7 & 15.00 & -- & -- & 15.12 & 25.84 & 0.40 & 7.21 & -- \\
\hline 69 & Neon & $0.80-0.99$ & 7 & 5.72 & 5.90 & 1.18 & 6.35 & 6.22 & 1.26 & 10.47 & 4.77 \\
\hline 70 & Argon & $0.82-0.99$ & 6 & 4.76 & 3.68 & 0.99 & 4.02 & 3.61 & 0.72 & 12.30 & 3.62 \\
\hline 71 & Carbon monoxide & $0.86-0.99$ & 5 & 9.51 & 4.57 & 1.06 & 10.59 & 4.67 & 1.23 & 13.05 & 3.20 \\
\hline 72 & Carbon dioxide & 0.87-0.99 & 4 & 3.65 & 15.04 & 0.93 & -- & -- & 0.29 & 12.26 & 5.83 \\
\hline 73 & Nitrogen & $0.85-0.99$ & 5 & 5.80 & 6.85 & 1.07 & -- & -- & 0.19 & 12.98 & 5.61 \\
\hline 74 & Fluorine & $0.80-0.99$ & 7 & 5.35 & 4.49 & 3.87 & 8.13 & 8.49 & 3.90 & \begin{tabular}{|l|}
7.10 \\
\end{tabular} & 4.29 \\
\hline 75 & Oxygen & $0.85-0.99$ & 5 & 4.64 & 6.45 & 0.29 & 1.22 & 15.82 & 0.36 & 12.67 & 5.77 \\
\hline 76 & Sulfur dioxide & $0.85-0.99$ & 5 & 10.43 & 13.56 & 0.20 & 19.71 & 10.00 & 1.42 & 13.54 & 3.76 \\
\hline \multirow[t]{2}{*}{77} & Chlorine & 0.84-0.99 & 6 & 5.99 & 13.69 & 0.99 & 29.95 & 38.59 & 0.38 & 10.39 & 9.50 \\
\hline & Average & & & 3.16 & 8.25 & 0.98 & 3.44 & 9.85 & 1.39 & 14.12 & 3.53 \\
\hline
\end{tabular}

*AARD $=\sum\left|\left(X_{\text {exp }}-X_{c a l}\right) / X_{\exp }\right| / N_{p} \times 100, \mathrm{X}=p^{\mathrm{s}}, v^{l}, v^{\mathrm{v}} ; N_{\mathrm{p}}$ : number of data points.

Together with the constraints of critical conditions $\left[\frac{\partial P}{\partial V}\right]_{T=T_{c}}=0,\left[\frac{\partial^{2} P}{\partial V^{2}}\right]_{T=T c}=0$ in the process of determination of the parameters, vapor pressure predictions accuracy of these EOS in nearcritical point region are all acceptable. As shown in Table 1, the average absolute relative deviations (AARD) between experimental and calculated saturated vapor pressure were 0.90, 1.33, 0.86, 0.98, and 1.39 for YSL, SRK, PR, CCOR, and PT, respectively. Trebble [18] drew some conclusions from a systematic review of eleven cubic equations including the cubic EOS that used in this work. The AARD given by Trebble and Bishnoi were SRK: AARD $p>1.4$, PR: AARD $p>1.39$, PT: AARD $p>1.30$, CCOR: AARD $p>2.06$. The calculated results in this work agree with that given by Trebble and Bishnoi in general. However, the AARD given in this work is slightly smaller than that given by Trebble and Bishnoi, because the temperature range is in this work is much smaller and closer to critical points. Figure 1 shows the saturated vapor pressure prediction deviation of methane given by the five equations. Obviously, we can see that the AARD at $T_{r}=0.7$ and $T_{r}=1.0$ is very small which agree with the introduction above. 


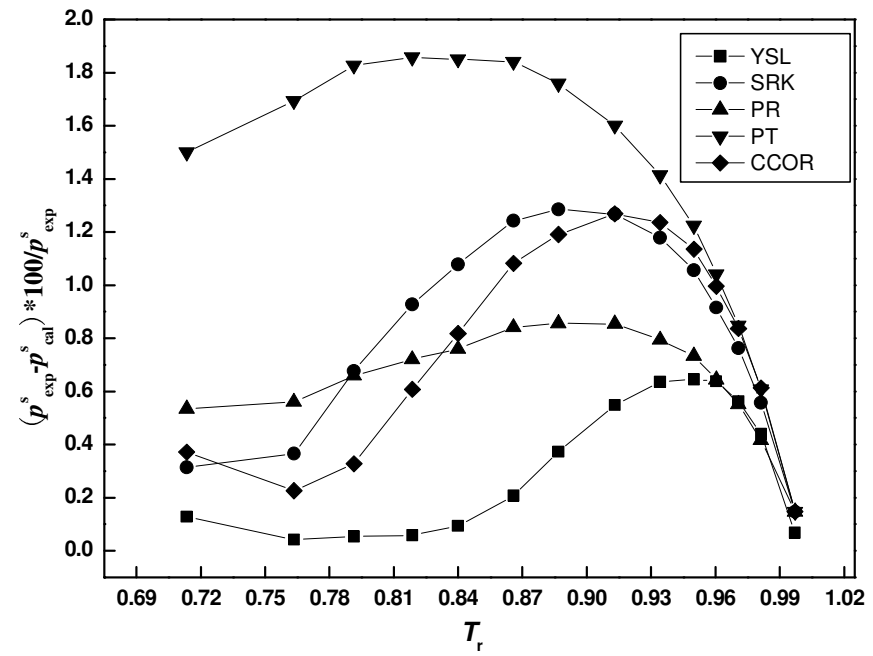

Figure 1. Saturated vapor pressure prediction deviation of methane.

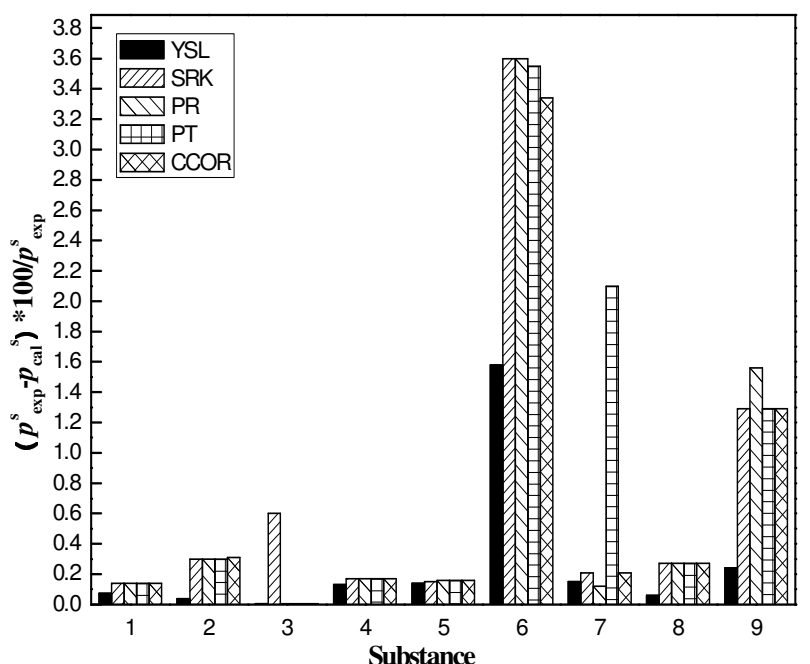

Figure 2. Deviations of calculated saturated vapor pressure at $T_{\mathrm{r}}=0.99$ 1: methane, 2: 3-ethyl-2methylpentan, 3: cyclopropane, 4: cyclopentane, 5: cycloheptane, 6: cycloheptane, 7: ethane, 8: propene, 9: neon.

\section{Comparison of saturated liquid molar volume}

As generalized above, lots of EOS parameters were evaluated by minimizing saturated vapor pressure deviation from experimental values and satisfying the equilibrium condition simultaneously, such as SRK and PR EOS. However, it is also apparent in the classic cubic EOS that improvements in saturated vapor volume predictions have often been obtained at the expense of liquid volume and pressure predictions and vice versa. The calculated results of saturated vapor and liquid molar volumes were also listed in Table 1. 


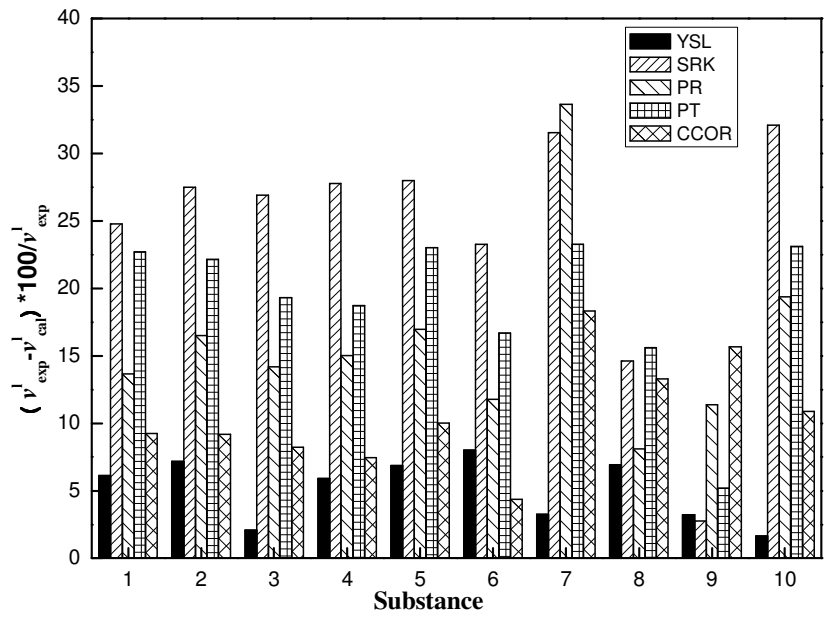

Figure 3. Deviations of calculated saturated liquid molar volume at $T_{r}=0.99$ 1: methane, 2 : ethane, 3: propane, 4: cyclopentane, 5: ethene, 6: propene, 7: ammonia, 8: CO, 9: neon, 10: $\mathrm{CCl}_{4}$.

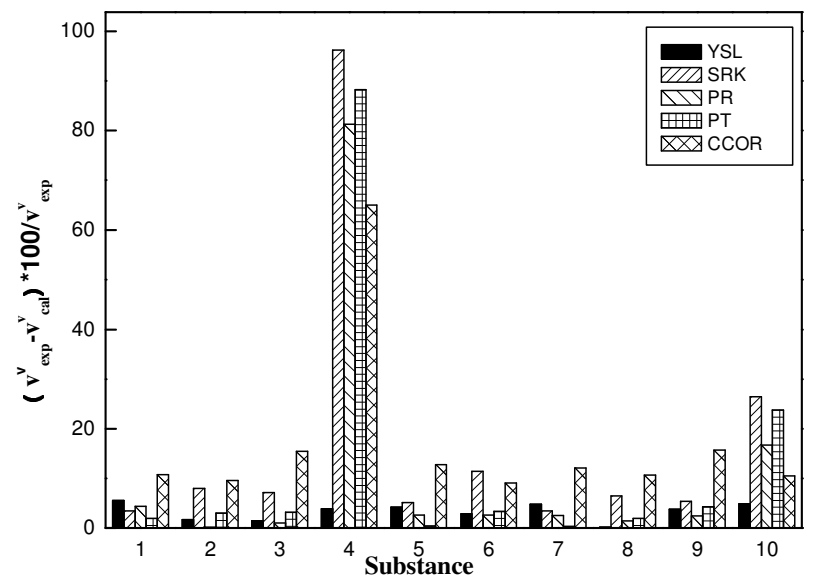

Figure 4. Deviations of calculated saturated vapor molar volume $T_{r}=0.99,1$ : methane, 2: propane, 3: 3-ethyl-2-methylpentan, 4: cyclopropane, 5: cyclopentane, 6: cycloheptane, 7: ethane, 8: propene, 9: benene, 10: neon.

The AARD between experimental and calculated saturated vapor molar volume given by Trebble [18] were 5.81, 5.34, 5.08, and 10.62 for SRK, PR, CCOR, and PT, respectively, and the AARD for saturated liquid molar volume were 17.64, 8.58, 6.78, and 4.60. Compared with the results listed in Table 1, the deviations shown by Trebble are much smaller than those in this work. As introduced above, this is because of the difference in calculation temperature range. Different from vapor pressure prediction accuracy which becomes better near the critical point, the volume prediction accuracy is much poor near critical point. Especially for SRK EOS, the prediction deviation of saturated liquid molar volume was up to $38 \%$ for some long chain hydrocarbons when critical points were approached. As noticed in this work, PT EOS has a good prediction far from critical point, mainly because it employed two component dependent 
constants for correlation, where one of the constants, which makes PT EOS possible to reproduce the critical point, is the pseudo critical compressibility factor. To compensate for this shortcoming, an artificial temperature-segment function $\left(0.9<T_{r}<=1\right)$ is introduced to force the pseudo critical back to $Z_{c}$, which leads to the discontinuity of some thermodynamic properties at $T_{r}=0.9$. The compensation is especially conspicuous for liquid volume. Compared to the four cubic EOS, the YSL quartic EOS preformed well not only in vapor pressure and vapor volume prediction, but also in liquid volume prediction. The AARD for the 77 substances calculated in this work was 2.07 for saturated liquid volume prediction, while this value was much bigger for the cubic EOS. Figures 3 and 4 showed deviations of calculated saturated liquid, and vapor molar volume at $T_{r}=0.99$. From the Figure 4 , we can see that the prediction accuracy of saturated vapor volume of cyclopropane at $T_{r}=0.99$ by the cubic EOS is very bad and unacceptable, while the result given by the YSL EOS is acceptable. This, on one hand, indicates the YSL EOS the great superiority over the cubic EOS used in this work. On the other hand, this may be caused by different data sources which used in regression of parameters of the EOS. Because there is tension in cyclopropane system, and cycloalkane of three carbon atoms is rather unstable, difference in experimental data may be resulted when temperature is close to critical point.

\section{CONCLUSIONS}

All the five EOS evaluated in this study can be expected to give fairly reliable predictions of saturated vapor pressures of the substances studied near critical points as well as far from them. Due to the oversimplified form, cubic EOS cannot describe simultaneously all properties accurately. The YSL quartic EOS has been found to be significantly superior to the others in both vapor pressure and saturated volume prediction in the vicinity of the critical point.

\section{ACKNOWLEDGMENTS}

This work was supported by the Zhejiang Provincial Natural Science Foundation of China (grant Y4110026) and Youth Foundation of Taizhou University (grant 2010QNBS11).

\section{REFERENCES}

1. Redlich, O.; Kwong, N.S. Chem. Rev. 1949, 44, 233.

2. Soave, G. Chem. Eng. Sci. 1972, 27, 1197.

3. Peng, D.Y.; Robinson, D.B. Ind. Eng. Chem. Fundam. 1976, 15, 59.

4. Schmidt, G.; Wenzel, H. Chem. Eng. Sci. 1980, 35, 1503.

5. Duan, Z.; Weare J.H. Geochim. Cosmochim. Acta 2000, 64, 1069.

6. Kiselev, S.B; Ely, J.F. Fluid Phase Equilibra 2004, 222, 149.

7. Lee, S.; Jeon, J.; Kim, W.; Chair, T.S. J. Phys. Chem. B 2008, 112, 15725.

8. Martin, J.J. Ind. Eng. Chem. Fund. 1979, 18, 81.

9. William, L.; Kubic, J. Fluid Phase Equilibra 1986, 31, 35.

10. Soave, G. Fluid Phase Equilibra 1990, 56, 39.

11. Shah, V.M.; Bienkowski, P.R; Cochran, H.D. AIChE J. 1994, 40, 152.

12. Lin, Y.L.; Bienkowski, P.R.; Shan, V.M.; Cochran, H.D. AIChE J. 1996, 42, 562.

13. Yun, Z.; Shi, M.R.; Shi; J.; Lee, H. Fluid Phase Equilibra 2001, 187-188, 275.

14. Kim, H.; Lin, H.M; Chao, K.C. Ind. Eng. Chem. Fund. 1986, 25, 75.

15. Patel, N.C.; Teja, A.S. Chem. Eng. Sci. 1982, 37, 463.

16. Smith, B.D.; Srivastava, R. Thermodynamic Data for Pure Compounds, Part A and Part B, Elsevier: Amsterdam; 1986.

17. Vargaftik, N.B. Tables on the Thermophysical Properties of Lquids and Gases, Hemisphere: Washington, DC; 1975.

18. Trebble, M.A.; Bishnoi, P.R. Fluid Phase Equilibria 1986, 29, 465.

Bull. Chem. Soc. Ethiop. 2011, 25(2) 